\title{
Uso de anillos y esmalte de uñas ¿Normar o no normar?
}

\author{
Rings and nail polish. Establish rules or not establish?
}

Mauro Orsini ${ }^{1}$ y Fernando Otaíza ${ }^{1}$

1Programa de Control de infecciones Asociadas a la Atención en Salud. Departamento de Calidad y Seguridad de la Atención. División de Gestión de la Red Asistencial. Ministerio de Salud de Chile.

Recibido: 12 de marzo de 2019

$\mathrm{E}$ n la presente edición de la Revista Chilena de Infectología se presenta un artículo que revisa el efecto del esmalte de uñas y uso de anillos sobre la efectividad de la higiene de manos como medida de prevención de infecciones. Como conclusión de esta revisión definida por sus autores como "no sistemática", se plantea la recomendación de restringir el uso de anillos y esmalte de uñas en la atención en salud bajo la premisa que "cualquier conducta que pueda disminuir la efectividad de la higiene de manos o constituir potencial riesgo para el paciente debiera evitarse, más aún cuando son prácticas sencillas de instaurar".

La medicina basada en la evidencia (MBE) y los métodos de evaluación de evidencia son herramientas que permiten no sólo informar sobre la magnitud de los beneficios o daños esperados al implementar o no una práctica, sino que la certeza que tenemos de la información en que se fundamenta la recomendación ${ }^{1}$, aun cuando no sea el único criterio a considerar para tomar la decisión. Si bien la MBE reconoce la existencia de distintos tipos de pruebas, enfatiza el uso de información proveniente de la investigación (especialmente investigación clínica), porque es este el tipo de evidencia que puede ser sometida al análisis crítico sobre su validez, efectividad y aplicabilidad ${ }^{2}$. Este es el rol de las revisiones sistemáticas que, a diferencia de otras metodologías, permite sistematizar la información disponible y evitar sesgos en su selección e interpretación por parte de los autores que en ocasiones lleva a seleccionar sólo artículos con evidencia favorable para la posición de los revisores o acomodar la interpretación de los datos con este mismo propósito. A modo de ejemplo, de cuatro revisiones sistemáticas que evaluaron el efecto de anillos en las manos del personal de salud, tres reconocen que la mala calidad de la evidencia existente es insuficiente para afirmar que el uso de anillos en los dedos aumente la presencia de bacterias en las manos del personal de salud o se asocie a mayor transmisión de microorganismos o mayor incidencia de infecciones en general ${ }^{3,4}$ o quirúrgicas en particular ${ }^{5}$, que en esta última se evaluó además el efecto del esmalte de uñas, con conclusiones similares. La cuarta revisión concluye que, reconociendo que la evidencia disponible es de baja calidad, en el ámbito quirúrgico, el uso de anillos no se asocia a mayor riesgo de infecciones de la herida operatoria ${ }^{6}$. No se encontraron revisiones sistemáticas que digan lo contrario.

El efecto de esmaltes de uñas y anillos sobre la efectividad de la higiene de manos y sus consecuencias sobre la incidencia de infecciones es un tema no resuelto a la fecha. Organismos internacionales como los CDC de E.U.A. ${ }^{7}$ y la Organización Mundial de la Salud ${ }^{8}$, en sus respectivas guías de higiene de manos, tras analizar la evidencia disponible, se abstienen de hacer recomendaciones específicas en ambos aspectos, indicando que "cada establecimiento de salud debiera desarrollar politicas sobre el uso de joyas y esmalte de uñas por el personal de salud. Estas politicas deben considerar el riesgo de transmisión de infecciones a pacientes y personal de salud, más que preferencias culturales". En esa declaración destaca la importancia que dan esas agencias a considerar la evidencia por sobre las creencias para normar o recomendar localmente las restricciones del uso de anillos o esmalte de uñas en el personal de salud. Por otra parte, el National Health Service de Inglaterra y varios países de la mancomunidad de naciones (Commonwealth) han adoptado políticas drásticas al respecto, como normar la práctica de "bare below the elbows" ("desnudo bajo los codos") que implica no usar joyas, relojes o ropa, en las manos y brazos desde los codos, además de requerir uñas sin esmalte, aunque permite el uso de argollas planas ${ }^{9}$ como son las argollas matrimoniales que algunos usan, 
aunque esta decisión no ha estado exenta de controversias y discusiones por la pobre evidencia que la respalda ${ }^{10-19}$. Independiente de lo anterior, hay consenso de los expertos en que el uso de anillos o esmalte de uñas no debe dificultar la higiene de manos y debe permitir evaluar si se hizo adecuadamente.

A diferencia de recomendar prácticas, que son propuestas no obligatorias, normar una práctica, sea de manera positiva (indicando que se debe realizar) o negativa (prohibiendo su uso), corresponde a una medida que conlleva consecuencias administrativas e incluso penales. Por ese motivo, la decisión sobre qué normar o no normar se debe fundamentar en criterios precisos, considerando las características del problema, sus consecuencias en términos de magnitud y gravedad, intervenciones disponibles, aspectos económicos, éticos, preferencias de los pacientes, cultura y otras regulaciones vigentes que incidan en la materia. Para normar sobre el tema es necesario contar con información que documente mayor frecuencia de fallas de la higiene de manos con el uso de anillos o esmalte comparado con no usarlos, y que esta falla se asocie a transmisión de agentes patógenos o a mayor incidencia de infecciones en situaciones endémicas o epidémicas, antecedentes que a la fecha no se han documentado.

\section{Referencias bibliográficas}

1.- Neumann I, Pantoja T, Peñaloza B, Cifuentes L, Rada G. El sistema GRADE: un cambio en la forma de evaluar la calidad de la evidencia y la fuerza de recomendaciones. Rev Med Chil 2014; 142: 630-5. doi: 10.4067/S003498872014000500012 .

2.- Biagini L. La evidencia y el proceso de toma de decisiones. Rev Med Chile 2005; 133: 871-3.

3.- Dyar A, Dyar O J. P 156: Ring wearing in healthcare settings: an evidence-based update. Antimicrob Resist Infect Control 2013; 2(Suppl 1): P156. doi:10.1186/2047-2994-2-S1-P156.

4.- Patel A. Impact of finger rings on the presence of bacteria on healthcare providers' hands. Thesis, Georgia State University, 2017. Disponible en: https://scholarworks.gsu.edu/ iph_theses $/ 560$.

5.- Arrowsmith V A, Taylor R. Removal of nail polish and finger rings to prevent surgical infection. Cochrane Database Syst Rev 2014; 8 : CD003325. doi: 10.1002/14651858.CD003325. pub3.

6.- Francis R H, Mudery J A, Tran P, Howe C, Jacob A. The case for using evidence-based guidelines in setting hospital and public health policy. Front Surg 2016; 3(March). doi: 10.3389 /fsurg.2016.00020.

7.- Centers for Disease Control and Prevention. Guideline for Hand Hygiene in Health-Care Settings: Recommendations of the Healthcare Infection Control Practices Advisory
Committee and the HICPAC/SHEA/APIC/ IDSA Hand Hygiene Task Force. MMWR 2002; 51: RR-16. Disponible en: www.cdc.gov/ $\mathrm{mmwr} / \mathrm{PDF} / \mathrm{rr} / \mathrm{rr} 5116 . \mathrm{pdf}$.

8.- World Health Organization. WHO Guidelines on Hand Hygiene in Health Care: First Global Patient Safety Challenge Clean Care Is Safer Care. Geneva, Switzerland: World Health Organization 2009: 270 p. Disponible en: https://apps.who.int/iris/bitstream/ handle/10665/44102/9789241597906_eng. pdf;sequence $=1$.

9.- $\mathrm{DH} / \mathrm{CNO}$ Directorate/Patient Environment Team. Uniforms and workwear: Guidance on uniform and workwear policies for NHS employers. Department of Health. 2010. Disponible en: https:// webarchive.nationalarchives.gov. uk/20130123201551/http://www.dh.gov.uk/ en/Publicationsandstatistics/Publications/ PublicationsPolicyAndGuidance/DH_114751.

10.- Magos A, Maclean A, Baker D, Goddard N, Ogunbiyi O. Bare below the elbows: A cheap soundbite. BMJ 2007; 335 (7622): 684.

11.- Horwitz MD, Sorene ED. Bare below the elbows policies: unnecessary bureaucracy. $\mathrm{Br} \mathrm{J}$ Hosp Med (Lond) 2010; 71:124-5.

12.- Willis-Owen CA, Subramanian P, Kumari P, Houlihan-Burne D. Effects of "bare below the elbows" policy on hand contamination of 92 hospital doctors in a district general hospital. J Hosp Infect 2010; 75:116-9. doi: 10.1016/j. jhin.2009.12.013.
13.- Burger A, Wijewardena C, Clayson S, Greatorex R A. Bare below elbows: does this policy affect handwashing efficacy and reduce bacterial colonisation? Ann R Coll Surg Engl 2011; 93: 13-6. doi: 10.1308/003588410X1277 1863936882 .

14.- Jones J, Shanks A. Laid bare: religious intolerance within online commentary about "bare below the elbows" guidance in professional journals. Health Care Anal 2013; 21: 271-81. doi: 10.1007/s10728-0130248-1.

15.- Pellerin J, Bearman G, Sorah J, Sanogo K, Stevens M, Edmond M B. Healthcare worker perception of bare below the elbows: readiness for change? Infect Control Hosp Epidemiol 2014; 35: 740-2. doi: 10.1086/676422.

16.- Henderson J, McCracken S. Bare below the elbows: Clinical value of a wristwatch. BMJ 2008; 336(7634):10. doi: 10.1136/ bmj.39423.586331.1F.

17.- Mcgoldrick M. Bare below the elbows: preventing cross contamination via the staff's attire. Home Healthc Nurse 2014; 32: 440-1. doi:10.1097/NHH.0000000000000112.

18.- Dancer S G, Duerden B I. Changes to clinician attire have done more harm than good. J R Coll Physicians Edinb 2014; 44: 293-8. doi: 10.4997/JRCPE.2014.410.

19.- Tse G, Withey S, Yeo J M, Chang C, Burd A. Bare below the elbows: was the target the white coat? J Hosp Infect 2015; 91: 299-301. doi: 10.1016/j.jhin.2015.08.003. 